\title{
Using a Dynamic Model to Simulate the Heuristic Evaluation of Usability
}

\author{
Nuria Hurtado ${ }^{1}$, Mercedes Ruiz ${ }^{1}$, and Jesús Torres ${ }^{2}$ \\ ${ }^{1}$ Department of Computer Languages and Systems \\ University of Cadiz \\ C/ Chile n ${ }^{\circ} 1$ - 11003 - Cadiz (Spain) \\ \{nuria.hurtado, mercedes.ruiz\}@uca.es \\ ${ }^{2}$ Department of Computer Languages and Systems \\ University of Seville \\ Avda. Reina Mercedes s/n - 41012 - Seville (Spain) \\ jtorres@lsi.us.es
}

\begin{abstract}
Among usability inspection methods, heuristic evaluation, or expert evaluation, is considered the most used and well-known usability evaluation method. The number of evaluators and their expertise are essential aspects that affect the quality of the evaluation, the cost that its application generates, and the time that it is necessary to spend. This paper presents a dynamic simulation model to analyze how different configurations of evaluator team have an effect upon the results of the heuristic evaluation method. One of the main advantages of using a dynamic simulation model is the possibility of trying out different decisions before carrying them out, and change them during the simulation of the evaluation process.
\end{abstract}

Keywords: Usability, usability evaluation, heuristic evaluation, dynamic simulation modeling.

\section{Introduction}

Heuristic evaluation or expert evaluation of usability is one of the most used and wellknown usability evaluation methods. It was originally developed by Nielsen and Molich [6] and it is possible to find numerous references about this evaluation method and how to carry it out [1][4][9]. The number and expertise of evaluators are essential aspects that affect the evaluation results in a heuristic evaluation. Knowing both, the number and expertise, it is possible to estimate the number of problems that can be found using the mathematical model presented by [8]. These two factors also affect the costs that the method incurs. But there is an additional key factor: time.

Using dynamic modelling and simulation will also allow us to obtain final values of these key factors, but the additional advantage is that it allows us to have visibility over time during the application of usability evaluation. Thus, it is possible to experience different decisions before carrying them out, as well as to introduce changes in the key input parameters during the simulation process. The simulation model can 
provide information about the results of the method when combinations of evaluators with different levels of expertise and dedication are used, thus allowing us to customize the model to the reality of an organization or a specific project.

This paper presents a dynamic simulation model to analyze the effects that the changes in number, expertise and dedication of evaluators have on the key factors: the quality of the evaluation results, the cost, and the time spent.

\section{Dynamic Simulation Model of Heuristic Evaluation}

The scheme proposed by Kellner et al. [3] and the methodology for model building by Martinez and Richardson [5] have been followed to build this model:

1. Model Purpose and Model Scope: The purpose of the model determines key questions to address, as well as the model scope. The purpose of the developed model is to analyze how the changes in number, expertise and dedication of evaluators have an effect upon the behaviour of the method over time. This effect will be assessed through time, cost and quality indicators. The scope of this model, in this case, is limited to modelling the behaviour of the problem detection phase of the heuristic evaluation method, which results in a single set of detected usability problems.

2. Output variables and Input Parameters: The output variables are the information items that must be known in order to be able to answer the key questions for which the model was created. In the model developed these indicators will be represented through the evolution of the following main output variables over time:

Usability problems found: This one represents the number of different usability problems found after analysis and aggregation of independent evaluations. It will hence determine evaluation quality.

Accumulated variable cost: This one represents the variable cost implied by implementing the method.

Time: The behaviour of time will be influenced by the dynamic nature of the simulation model.

The main input parameters of the model will make it possible to configure different simulation scenario:

Number of evaluators $E(i)$ : this parameter is subdivided into three other parameters depending on the level of expertise, making it possible, as well, to change their time dedication, hence variation in evaluator team composition is allowed for during simulation. E1: It represents the number of novice evaluators (level 1). E2: It represents the number of evaluators with expertise in usability (level 2). E3: It represents the number of evaluators with expertise in usability as well as in the mastering of the specific system (level 3).

Usability problems to be found: This one represents the total number of estimated usability problems depending on the size and type of system to be evaluated.

Single evaluation capacity $E(i)$ : This parameter is also subdivided into E1, E2, and E3 and it will represent the amount of usability problems that an evaluator can sort out per time unit (hour).

Single cost $E(i)$ : This parameter is also subdivided into E1, E2, and E3 and it represents the cost (in Euro) per time unit (hour) of an evaluator. 
Single problems proportion $E(i)$ : This parameter is also subdivided into E1, E2, and E3 and it represents the average rate of problems that a single evaluator may be able to find. It may be estimated using Nielsen's mathematical model [8] or obtained from other similar studies, or from previous project metrics collections.

3. Model Conceptualization and Model Formulation: The concepts involved in a system dynamics model are normally represented in a causal loop diagram. In this step we have used casual loop diagrams to collect the cause and effect relationships found in the heuristic evaluation method. Once the model has been conceptualized, its formalization as a mathematical model follows. We have used stock and flow diagrams [2] in this step since they help us to represent the structure of the model and provide a bridge to simulation modelling by facilitating the assignment of equations to this structure and the identification of relationships between the variables involved. These diagrams are made up by three main elements: level variables, flow variables, and auxiliary variables. In the model developed three level variables have been considered: usability problems found, accumulated variable cost and usability problems to be found. Two flow variables have been added in the model: problem detection rate and cost increase rate. The first one represents the flow between usability problems to be found and usability problems found. The second one increases the level variable accumulated variable cost as the simulation progresses. Auxiliary variables are the rest of elements in the process that have an influence upon it. It is also necessary to have constants or input parameters that represent external influences on the model. Once the model has been developed and behaviour-governing equations have been introduced, simulation can be carried out by setting different scenarios.

4. Model Assessment: There are two main aspects that must be taken into account to carry out model assessment: model verification and model validation [10]. Model verification assesses that the model implementation is error free and, that it is a right representation of the intended logical behaviour of the system under study. Model validation assesses that the model helps solve the end user's problems within the context of study. We have carried out different tests to validate and verify the structure and behaviour of the model developed with satisfactory results.

5. Model Simulation and Analysis of Results: The model has been implemented using the $\operatorname{Vensim}^{\circledR}$ simulation environment. First of all, to simulate the model we have set the values for the main input parameters: usability problems to be found, problem single proportion, single capacity, and single variable cost. Data from various projects published in the related literature have been used to set up the base case of our study [7][9]. Then, the effect of the evaluators can be studied by setting several experiments with different scenarios, each one having different values for the input parameter: number of evaluators $E(i)$. For instance, it is possible to compare different scenarios in a simulation using different number of evaluators of the same level of expertise and dedication. In other instances, the simulation model can also provide information about the behaviour of the method when combinations of evaluators with different levels of expertise or dedication are used. The changes in the input parameters allow us to customize the model to the reality of an organization or a specific project. The simulation results of the different experiments carried out is represented in graphs of evolution over time for the result variables. The analysis of these graphs allows us to help decision making in the usability evaluation. 


\section{Conclusions}

This work illustrates the application of the modelling and simulation techniques to the heuristic evaluation method. The model has been conceptualized and formalized under the system dynamics approach. The implementation of the simulation model makes it possible to add visibility over time during the application of usability evaluation. It allows us to analyse the effect that changes in the number, expertise and dedication of evaluators have upon the results of cost, time and quality, making it possible to try out different policies to manage usability evaluation. The model provides a tool to help decision making for any organization that intends to implement usability evaluation methods. Our next tasks will be aimed at the development of simulation models of other usability evaluation methods.

Acknowledgements. The authors wish to acknowledge the Interdepartmental Commission for Science and Technology and the European Regional Development Fund (Spain), for subsidizing this research through projects TIN2007-67843-C06-03 and TIN2007-67843-C06-04.

\section{References}

1. Daly-Jones, O., Bevan, N., Thomas, C.: Handbook of User Centered Design. IE2016 INUSE Deliverable D6.2.1 Version 1.31. Serco Usability Services, National Physical Laboratory, Teddington, Middx, UK (2001)

2. Forrester, J.W.: World Dynamics. Productivity Press, Cambridge (1973)

3. Kellner, M.I., Madachy, R.J., Raffo, D.M.: Software Process Simulation Modeling: Why? What? How? The Journal of Systems and Software 46(2), 91-105 (1999)

4. Lindgaard, G.: Usability Testing and System Evaluation. Chapman \& Hall, London (1994)

5. Martinez, I.J., Richardson, G.P.: Best Practices in System Dynamics Modeling. In: Hines, H., Diker, V.G. (eds.) Plenary paper: Proceedings of the 29th International Conference of the System Dynamics Society, Atlanta, GA, USA (2001)

6. Nielsen, J., Molich, R.: Heuristic evaluation of user interfaces. In: Proceedings of the SIGCHI Conference on Human Factors in Computing Systems: Empowering People, Seattle, Washington, United States, pp. 249-256 (1990)

7. Nielsen, J.: Finding Usability Problems through Heuristic Evaluation. In: Proceedings of the SIGCHI Conference on Human Factors in Computing Systems, ACM CHI 1992, Monterey, CA, USA, pp. 373-380 (1992)

8. Nielsen, J., Landauer, T.K.: A Mathematical Model of the Finding of Usability problems. In: Proceedings of the ACM INTERCHI 1993 Conference, Amsterdam, The Netherlands, pp. 206-213 (1993)

9. Nielsen, J.: Heuristic Evaluation. In: Nielsen, J., Mack, R.L. (eds.) Usability Inspection Methods, pp. 25-62. John Wiley \& Sons, New York (1994)

10. Sterman, J.D.: Business Dynamics. In: Systems Thinking and Modeling for a Complex World. McGraw-Hill, New York (2000) 